\title{
Imaging Condition Optimization in Multiphoton Microscopy of Three-Dimensional Collagen Fiber Structures
}

\author{
Chih-Yuan Hsiao ( 蕭志遠), Yen Sun ${ }^{\mathrm{b}}$ ( 孫 嬿), Wen Lo ( 駱 文 ), \\ Sung-Jan Lin ${ }^{\mathrm{c}}$ ( 林頌然), Shiou-Hwa Jee ${ }^{\mathrm{c}, \mathrm{d}}$ ( 紀秀華 ), \\ Gwo-Jen Jan ${ }^{\mathrm{a}}$ ( 詹國偵) and Chen-Yuan Dong ${ }^{\mathrm{b} *}$ ( 董成淵) \\ anstitute of Electro Optics, Department of Electrical Engineering, National Taiwan University, \\ Taipei 106, Taiwan, R.O.C. \\ ${ }^{\mathrm{b}}$ Department of Physics, National Taiwan University, Taipei 106, Taiwan, R.O.C. \\ ${ }^{\mathrm{c}}$ Department of Dermatology, National Taiwan University Hospital, Taipei 100, Taiwan, R.O.C. \\ ${ }^{\mathrm{d}}$ Department of Dermatology, National Taiwan University College of Medicine, Taipei 100, Taiwan, R.O.C.
}

\begin{abstract}
Multiphoton generated autofluorescence and second harmonic signals are useful luminescent signatures for probing the structural and spectral properties of collagen fibers. However, optimization of image formation deep within a three-dimensional collagen sample may be hindered by index-mismatch induced spherical aberration. In this work, we examined this issue by comparing the axial multiphoton luminescence profiles of bovine tendon under water, glycerin, and oil immersion conditions. Our results show that, although in general, oil immersion imaging resulted in the slowest decay of measured axial luminescence profile, other immersion conditions can perform better at specific locations within the tendon, indicating the existence of local variations of refractive indices within the collagen-filled tendon. Our results suggest that optimizing multiphoton imaging of collagen requires acquiring and comparing imaging results using different immersion fluids and that the results may be used to infer the spatial distribution of refractive indices in biological specimens.
\end{abstract}

Keywords: Multiphoton microscopy; Second harmonic; Fluorescence; Collagen; Spherical aberration.

\section{INTRODUCTION}

\section{Multiphoton excitation microscopy}

In the decade since the development of two-photon fluorescence microscopy, microscopic imaging using multiphoton excitation has developed into one of the most important techniques in modern optical microscopy. ${ }^{1}$ Compared to the widely used one-photon excitation microscopy, the multiphoton modality offers a number of distinct advantages. First, the non-linear nature of the excitation probability limits sample excitation to the focal volume and optical scanning with the point-like excitation volume results in confocal-like images without the use of confocal apertures. Furthermore, the near-infrared photons used for non-linear excitation are absorbed and scattered less by tissue components than the UV or visible photons used in one-photon fluorescence microscopy. The enhanced depth penetration of multiphoton microscopy allows in-depth, three-dimensional imaging without invasive histological procedures. This feature is particularly important for in vivo tissue imaging. Finally, the wide spectral separation between the multiphoton excitation wavelengths and the fluorescence emission allows easy detection of the entire emission spectra. ${ }^{2}$ The unique features of multiphoton microscopy allow it to be applied in addressing important issues in a number of life science disciplines such as neurobiology, developmental biology, deep-tissue imaging, and transdermal drug delivery. ${ }^{3-6}$

\section{Multiphoton imaging of collagen}

In multiphoton imaging, a particular tissue structure that has attracted considerable attention is collagen fibers. The different types of collagen serve important structural roles in human physiology. For example, type I collagen exists in great abundance in structures such as the skin dermis and the tendons while type II collagen is the major component of the cartilage. ${ }^{7}$ The unique physiological roles of collagen enable it to become useful biomaterial for life science applications. For example, collagen has been used as a scaffold for nerve regeneration and drug delivery systems. ${ }^{8}$ In these applications, it may be advantageous to be able to follow the 
structure of collagen and surrounding materials as a function of time. The enhanced imaging depth of multiphoton microscopy is the ideal technique for evaluating collagen-based biotechnological products. Collagen luminescence has been extensively studied under multiphoton microscopy. ${ }^{9,10,11}$ Depending upon the excitation wavelength used, collagen fibers can emit varying degrees of autofluorescence and harmonic signals. ${ }^{9}$ It is clear that the ability to acquire three-dimensional, microscopic images of collagen structures is of great value in bioimaging.

Unlike conventional microscopic imaging techniques, multiphoton microscopy has the ability to image deep within the specimen. However, special considerations must be given in order to achieve in-depth, optimized imaging conditions of structurally heterogeneous specimens such as biological tissues. In particular, two factors which can adversely affect in-depth imaging are scattering and refractive-index mismatch induced spherical aberration. In the case of skin, optical coherence tomography (OCT) has demonstrated that the skin can be approximated as a layered structure varying in refractive indices and scattering coefficients. For the index of refraction, the surface stratum corneum has a value of about 1.47. With increasing depths, the refractive index decreases to 1.43 in the granular layer and continues to decrease to around 1.34 at the basal layer. Beyond the basal layer, there is an increase of the refractive index in the upper dermis to around 1.41. Scattering coefficients of the skin also display a complex dependence on depth. The scattering coefficients for the stratum corneum, granular layer, basal layer, and the upper dermis are $1-1.5,6-7,4-5$, and $5-8 \mathrm{~mm}^{-1}$, respectively. ${ }^{12,13}$ In less scattering tissue such as the cornea, the refractive indices of the stroma is a function of the degree of tissue hydration and can vary between approximately 1.35 and 1.38. ${ }^{14}$ The influences of scattering and refractive index in multiphoton image formation have been well studied. In general, it is accepted that while scattering can affect detected fluorescence in-depth, they do not contribute to resolution degradation. On the other hand, refractive index mismatch induced spherical aberration can lead to degraded resolution and detected fluorescence. ${ }^{15-17}$ Therefore, to optimize the imaging of any biological specimen, efforts need to be made to counter the effects of these two optical phenomena. In the case of spherical aberration, one possibility to optimize image formation is to vary the immersion medium of the microscope objective used for sample observation. This approach has been successfully applied to multiphoton skin imaging and it was found that an oil immersion objective outperformed a water lens. ${ }^{17}$ However, to the best of our knowledge, the technique of varying the objective immersion conditions has not been applied to collagen fiber imaging. Therefore, in this work, we acquired the multiphoton images of collagen fibers from bovine tendon under water, glycerin, and oil immersion conditions to better characterize and optimize collagen fiber imaging in 3-D.

\section{MATERIALS AND METHODS}

\section{Multiphoton excitation microscopy of bovine tendon}

The basic arrangement of the multiphoton microscope used in this study has been well described in the literature. ${ }^{17}$ In this apparatus, a diode-pumped (Millennia X, Spectra Physics, Mountain View, CA), femtosecond titanium-sapphire laser (Tsunami, Spectra Physics) is used as the excitation source. The $880 \mathrm{~nm}$ output of the titanium-sapphire laser, used as the multiphoton excitation source for collagen luminescence, is guided toward an upright microscope (E800, Nikon, Japan). Prior to entering the microscope, the laser beam is incident upon an $\mathrm{x}-\mathrm{y}$ scanning system (Model 6220, Cambridge Technology, Cambridge, MA). The scanning system then guides the reflected laser beam into the excitation path of the microscope. The excitation path is modified to include a pair of lenses for beam expansion. The beam expansion is necessary to ensure overfilling of the objective's back aperture for optimizing image resolution. The expanded laser beam is then reflected into the objective by a dichroic mirror (720DCSPXR, Chroma Technology, Brattleboro, VT). In this fashion, the average powers achieved at the samples are 3.2, 2.8 , and $2.8 \mathrm{~mW}$ under water, glycerin, and oil immersion conditions, respectively. The luminescence generated at the multiphoton focal spot is then collected by the same focusing objective, passed through the dichroic mirror, an additional short-pass filter (E680SP, Chroma Technology), and a bandpass filter centered at $440 \mathrm{~nm}$ (HQ440/40, Chroma Technology) prior to reaching the single photon counting photomultiplier tubes (R7400P, Hamamatsu, Japan). The dichroic mirror and filter combination is intended to collect the second harmonic generation ( $\mathrm{SHG}$ ) signal from the collagen fibers from the bovine tendon in the back-scattering geometry. In this manner, planar images composed of $256 \times 256$ pixels are collected. Axial sectioning of the specimen is achieved by moving the objective focal spot to different focusing depths. This can be achieved by a piezoelectric transducer or a motor-drive attached to the objective focusing control of the microscope. To investigate the effects of different immersion objectives on in-depth imaging of bovine tendon, we selected 
a multi-immersion objective (20x Plan Fluor, NA 0.75, WD $0.35 \mathrm{~mm}$, Nikon) equipped with a correction collar to be used under water, glycerin, and oil immersion conditions. The bovine tendon sample is easily prepared by mounting on a glass slide with a No. 1.5 cover glass separating the tendon from the objective.

\section{RESULTS AND DISCUSSIONS}

The multiphoton generated second harmonic signal of a section of the bovine tendon is shown in Figs. 2 and 3. Displayed in Fig. 2 is a 3-D reconstruction of the bovine tendon acquired under water immersion condition. We performed the SHG imaging of the same region of the bovine tendon under water, glycerin, and oil immersion conditions and the results are shown in Fig. 3. To compare the objective performances at different depths, SHG images from four depths $(0$, 30,54 , and $95 \mu \mathrm{m}$ ) were chosen for image comparison. In this study, the $0 \mu \mathrm{m}$ position is defined to be the surface of the tendon section while the other three depths are the mechanical distance the objective moved relative to the tendon surface. For the ease of comparison, at each depth, the images acquired under water, glycerin, and oil immersions are normalized. A qualitative comparison of Fig. 3 shows several important features. First, for all three immersion conditions, the image resolution appears to degrade with increasing imaging depths. Structurally, details of the collagen fibers appear to be the most discernible at the surface. With increasing imaging depths, the fiber structures become more blurry. In addition, the normalized SHG intensity of the images did not show significant differences for the three immersion conditions until at the imaging depth of $95 \mu \mathrm{m}$. At this depth, the SHG image acquired under the oil immersion condition was the most intense followed by the glycerin immersion result, and the water immersion image is the least intense of the three images. Since the same area within the bovine tendon

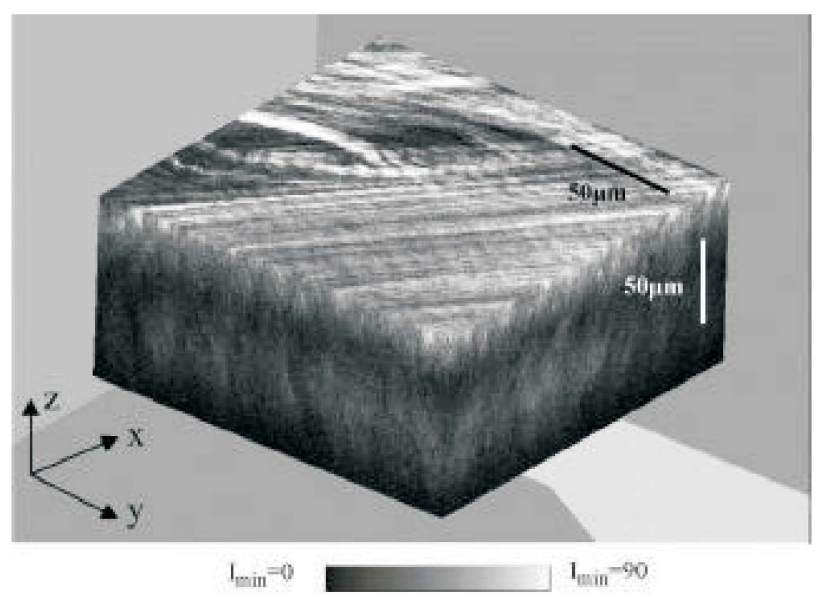

Fig. 2. Multiphoton image of a 3-D reconstructed bovine tendon acquired under water immersion condition.

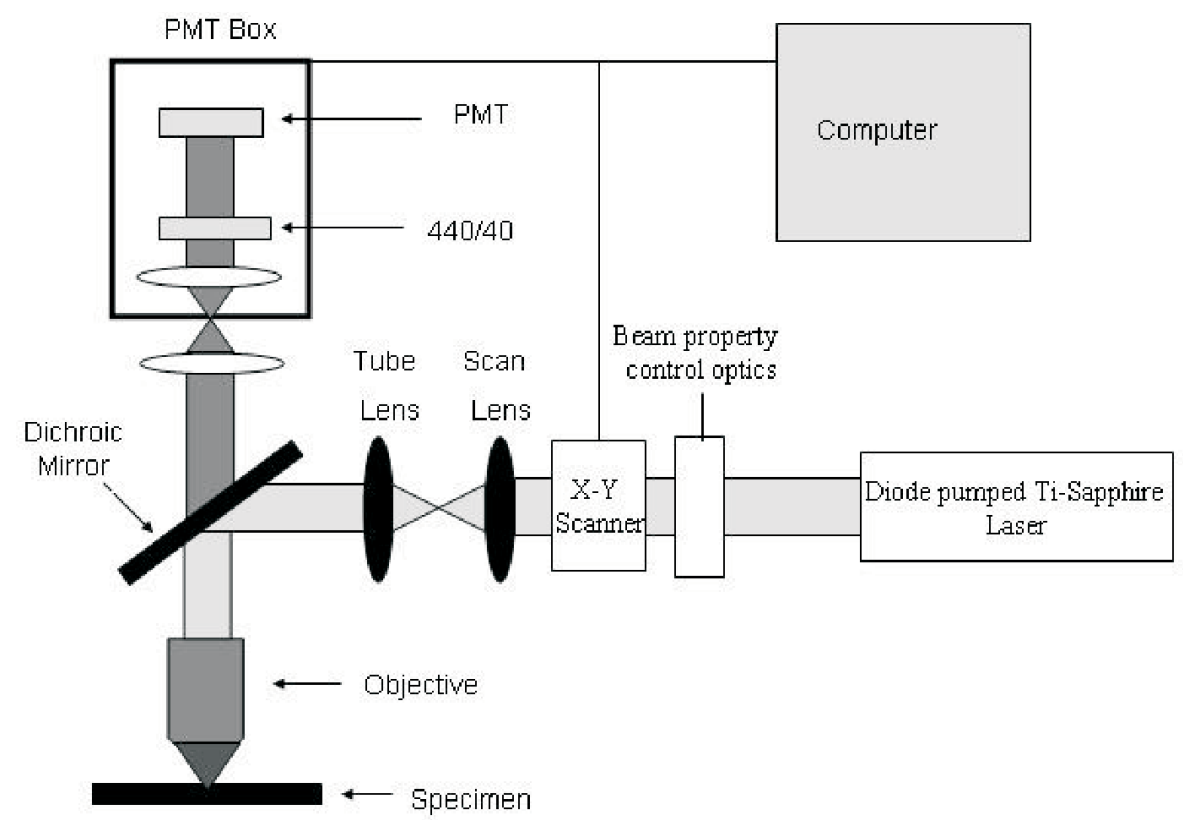

Fig. 1. The set-up of a multiphoton microscope. 
was imaged, our results support the fact that index mismatch induced spherical aberration is the main contributor to resolution degradation and the decrease in measured SHG intensity with depths.

To quantify our observations, we calculated the axial intensity profiles of the SHG signal for the images displayed in Figs. 2 and 3 and plotted the results in Fig. 4a. The axial intensity profile is obtained by averaging the SHG signal of the image (at a given depth) and the result is plotted at different depths. To compare the performance under different immersion conditions, we plotted the ratio of the average signal at each depth obtained under water and glycerin immersions to that measured using the oil immersion objective and the re- sult is shown in Fig. 4b. Figs. 4a and 4b shows an interesting result. Up to the imaging depth of approximately $40 \mu \mathrm{m}$, glycerin immersion achieved a higher, average SHG signal than that acquired under water immersion. However, the intensities under water and glycerin immersions are consistently lower than the oil immersion result, indicating that the average refractive index of this area of the tendon specimen is closer to that of immersion oil than glycerin or water. However, the depth profiles of the intensity are quite different at depths below $40 \mu \mathrm{m}$. At imaging depths between 40 and 60 $\mu \mathrm{m}$, the water-oil and glycerin-oil intensity ratios rose above 1 , with the water-oil ratio slightly above that of the glycerin-oil ratio. This observation suggests that this region of the
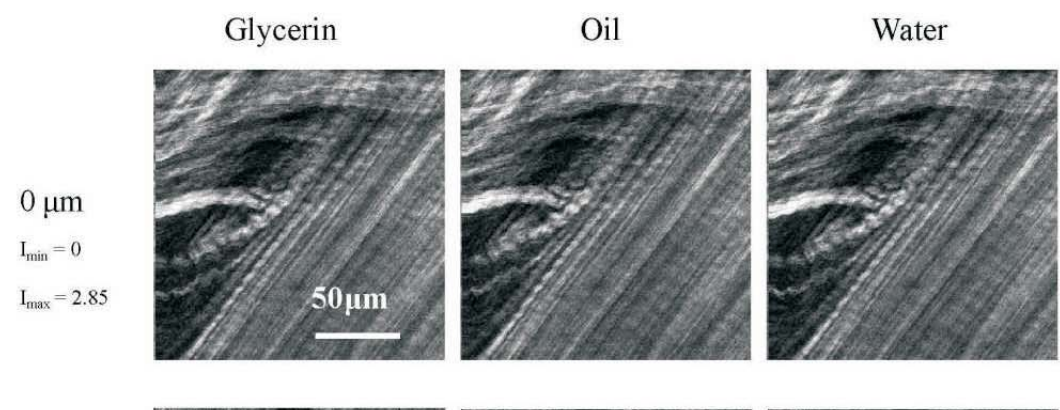

$30 \mu \mathrm{m}$
$\mathrm{I}_{\min }=0$
$\mathrm{I}_{\max }=1$
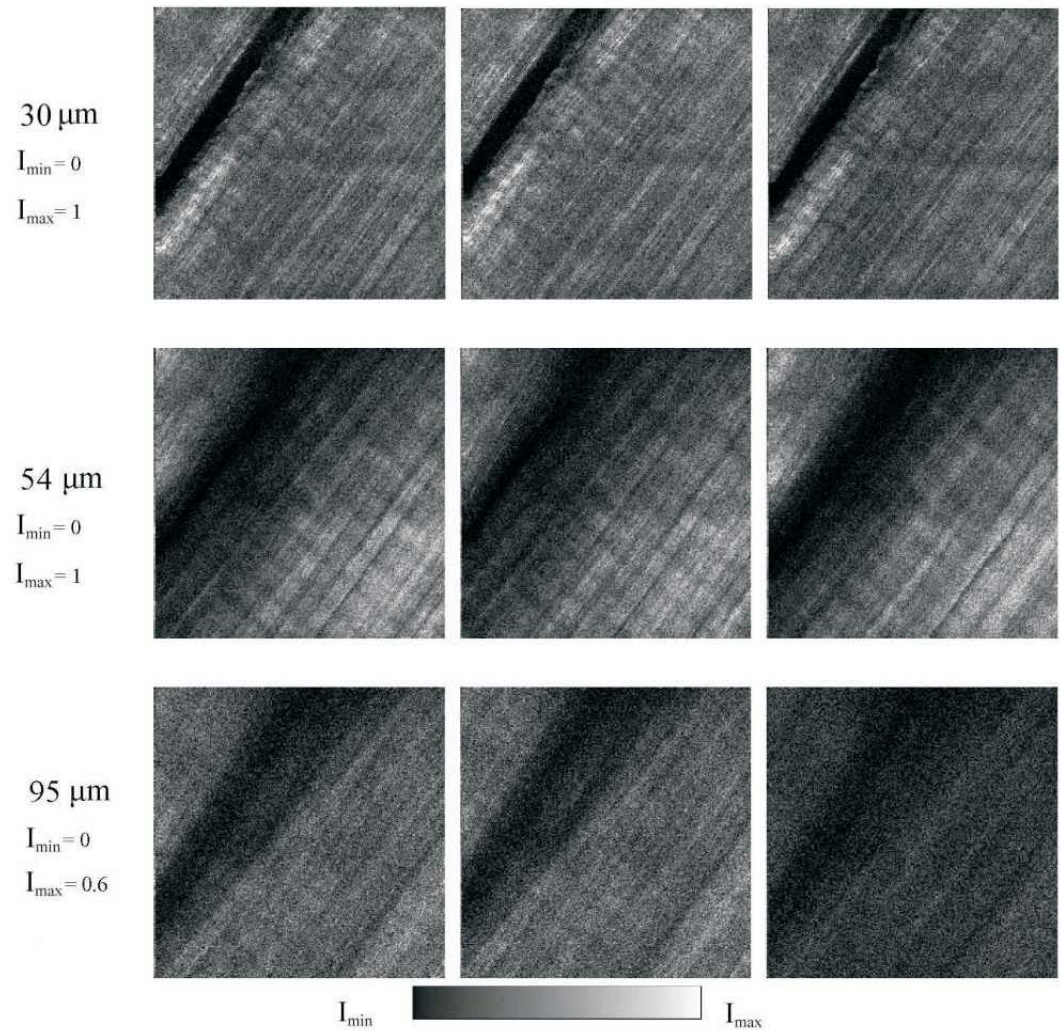

$I_{\max }$

Fig. 3. Cross sectional images of bovine tendon acquired under glycerin, oil, and water immersion conditions at different depths. 

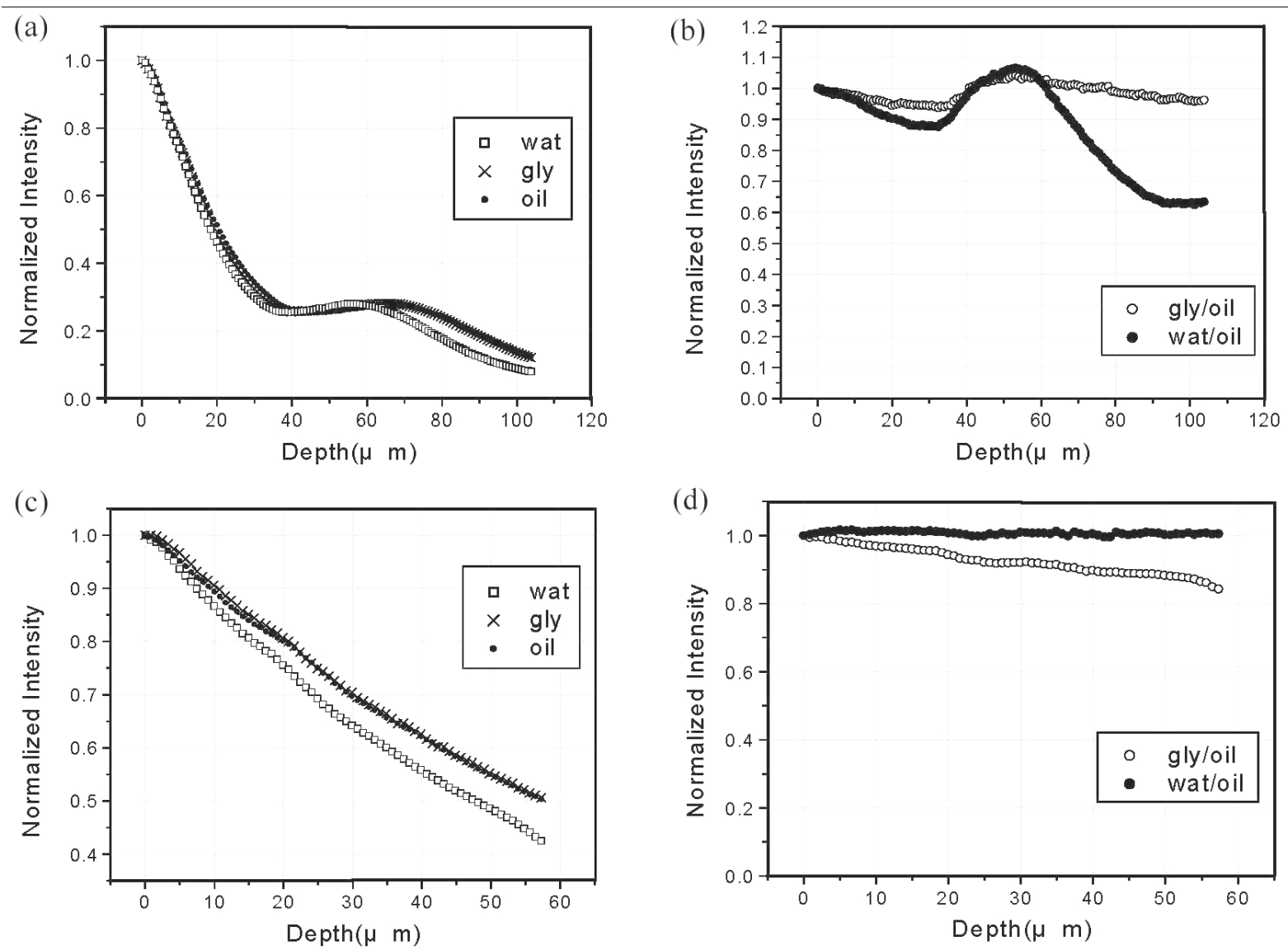

Fig. 4. (a) Normalized axial intensity profile of an area of bovine tendon (b) Normalized intensity ratios of the data acquired under the three immersion conditions (c), (d) are the corresponding figures for a different area of the bovine tendon.

tendon is not uniform in refractive indices. There appears to be a region at depths between 40 and $60 \mu \mathrm{m}$ where the refractive index of the tendon is closer to that of water than glycerin or oil. At imaging depths below $60 \mu \mathrm{m}$, the water-oil and glycerin-oil ratios again drop to below 1, indicative of the fact that, in this region, the tendon's refractive index is closer to that of oil than glycerin or water.

We also performed the SHG imaging of the tendon by acquiring the three-dimensional images of the specimen at a different position. For comparison, we only show the axial intensity and intensity ratio profiles in Figs. $4 \mathrm{c}$ and 4d. Figs. 4c and $4 \mathrm{~d}$ have a different behavior than that shown in Figs. $4 \mathrm{a}$ and $4 \mathrm{~b}$. Figs. $4 \mathrm{c}$ and $4 \mathrm{~d}$ show that the intensity acquired using the glycerin and oil immersion lenses match closely to each other while the water immersion result consistently measured a lower normalized intensity. This observation suggests that, in this region of the tendon, the average refractive index is between that of glycerin and oil and that it has a uniform distri- bution.

\section{CONCLUSION}

In this work, we investigated the multiphoton imaging of collagen filled bovine tendon under different immersion conditions. Three-dimensional, second harmonic images of the tendon specimen were acquired under water, glycerin, and oil immersion conditions. In addition to qualitative examination of the image qualities, we compare the average intensity acquired at different imaging depths. Our results suggest that despite the uniform appearance of tendon composition, the refractive index distribution within the tendon specimen is not spatially uniform. As a result, different immersion objectives may achieve optimal imaging at particular imaging depths. Our results suggest that variations in multiphoton generated, axial intensity distributions acquired under differ- 
ent immersion conditions may be a useful method for determining the refractive index distribution of biological specimens.

\section{ACKNOWLEDGMENT}

We acknowledge the support of NSC 92-2112-M-002018 and NSC 92-3112-B-002-048 from the National Science Council (Taiwan) for the completion of this project.

In addition, since returning to Taiwan, the author (C.Y.D.) has been under the support and guidance from Dr. Sunney I. Chan. Dr. Chan's scientific tenacity and persistent enthusiasm serve as solid examples for young scientists and C.Y.D. is grateful for having had the honor to work with Dr. Chan.

Received March 23, 2004

\section{REFERENCES}

1. Denk, W.; Strickler, J. H.; Webb, W. W. Science 1990, 248, 73.

2. So, P. T. C.; Dong, C. Y.; Masters, B. R.; Berland, K. M. Annu. Rev. Biomed. Eng. 2000, 2, 399.

3. Denk, W.; Sugimori, M.; Llinas, R. P. Natl. Acad. Sci. USA $\mathbf{1 9 9 5}, 92,8279$.
4. Squirrell, J. M.; Wokosin, D. L.; White, J. G.; Bavister, B. D. Nat. Biotechnol. 1999, 17, 763.

5. Dong, C. Y.; Yu, B.; Kaplan, P. D.; So, P. T. C. Microscopy Research and Technique 2004, 63, 81.

6. Yu, B.; Dong, C. Y.; So, P. T. C.; Blankschtein, D.; Langer, R. J. Invest Dermatol 2001, 117, 16.

7. Lodish, H.; Berk, A.; Zipursky, S. L.; Matsudaira, P.; Baltimore, D.; Darnell, J. E. Molecular Cell Biology; W. H. Freeman: New York, 2000; Chapter 22.

8. Yannas, I. V. In Biomaterials Science; Ratner, B. D.; Hoffman, A. S.; Schoen, F. J.; Lemons, J. E., Ed.; Academic Press: San Diego, 1996; pp 84-94.

9. Zoumi, A.; Yeh, A.; Tromberg, B. J. Proceedings of the National Academy of Sciences of the United States of America 2002, 99, 11014.

10. Stoller, P.; Reiser, K. M.; Celliers, P. M.; Rubenchik, A. M. Biophys. J. 2002, 82, 3330.

11. Yeh, A. T.; Nassif, N.; Zoumi, A.; Tromberg, B. J. Optics Letters 2002, 27, 2082.

12. Knuttel, A.; Boehlau-Godau, M. J. Biomed. Opt. 2000, $5,83$.

13. Tearney, G. J.; Brezinski, M. E.; Southern, J. F.; Bouma, B. E.; Hee, M. R.; Fujimoto, J. G. Opt. Lett. 1995, 20, 2258.

14. Meek, K. M.; Dennis, S.; Khan, S. Biophys. J. 2003, 85, 2205.

15. Dunn, A. K.; Wallace, V. P.; Coleno, M.; Berns, M. W.; Tromberg, B. J. Applied Optics 2000, 39, 1194.

16. Dong, C. Y.; Koenig, K.; So, P. T. C. Journal of Biomedical Optics 2003, 8, 450 .

17. Dong, C. Y.; Yu, B.; Kaplan, P. D.; So, P. T. C. Microscopy Research and Technique 2004, 63, 81. 